\title{
Clinical and neuroimaging profiles to identify C9orf72-FTD patients and serum Neurofilament to monitor the progression and the severity of the disease
}

\author{
Giulia Lucidi $^{1,2}$ | Valentina Berti ${ }^{3}$ | Irene Piaceri ${ }^{1,2}$ | Andrea Ginestroni ${ }^{1}$ | \\ Gemma Lombardi $^{1}$ | Camilla Ferrari ${ }^{1}$ | Cristina Polito ${ }^{3}$ | Valentina Bessi ${ }^{1}$ | \\ Alberto Pupi $^{4}$ | Benedetta Nacmias ${ }^{1,2}$ | Sandro Sorbi ${ }^{1,2}$ | Silvia Bagnoli ${ }^{1,2}$ (D)
}

\footnotetext{
${ }^{1}$ Department of Neuroscience, Psychology, Drug Research and Child Health, University of Florence, Florence, Italy

${ }^{2}$ IRCCS Fondazione Don Carlo Gnocchi, Florence, Italy

${ }^{3}$ Nuclear Medicine Unit, Department of Biomedical Experimental and Clinical Sciences "Mario Serio", University of Florence, Florence, Italy

${ }^{4}$ Foundation Turati, Pistoia, Italy
}

\section{Correspondence}

Silvia Bagnoli, Department of Neuroscience, Psychology, Drug Research and Child Health, University of Florence, Viale Pieraccini 6, 50139 Florence, Italy.

Email: bagnolis@unifi.it

Funding information

Italian Ministry of Health, Grant/Award Number: GR-2011-02347190; Fondo Ateneo 2017 Nacmias

\begin{abstract}
Background: The C9orf72 pathogenetic GGGGCC hexanucleotide repeat expansion is one of the most common causes of Frontotemporal Dementia (FTD), and early identification of this mutation is crucial for clinical and prognostic outcome. Although promising preclinical studies tried to evaluate the efficacy of biomarkers to identify C9orf72 expansion carriers, they have not been adequately assessed in humans.

Aim: To identify clinical, neuroimaging, and biological features that could be used to identify FTD subjects eligible to genetic testing.

Methods: This is a retrospective, case-control study of clinical, neuropsycological, biological, and neuroimaging data of C9orf72 expansion carriers compared with nonmutated FTD patients. Neurofilament light chain was evaluated by Quanterix Simoa essay.

Results: The study revealed a set of peculiar characteristics in patients with C9orf72 expansion, compared with not expanded, as: lower age at onset, higher number of familial form and affected relatives, higher FTD-MND symptoms, frequent behavioral and motor disorders at onset, higher incidence of dysphagia, lower mnesic deficits and higher rate of "Average" and "Rapid" progression of the disease. Neuroimaging data reveled that C9orf72 subgroup patients showed a higher hypometabolism of the frontal lobes involving both cortical and subcortical regions. Moreover, in symptomatic C9orf72 patients, NfL concentration was strongly elevated compared with presymptomatic carriers, increasing after 1 year of follow-up.

Conclusion: The metabolic signature combined with the clinical features could be a helpful tool to direct the genetic counseling. We confirmed that serum NfL evaluation could be a valid biomarker to monitor the disease severity and progression.

KEYWORDS

18FDG-PET, C9orf72, frontotemporal dementia, neurofilament light chain, neuropsychology
\end{abstract}




\section{1 | INTRODUCTION}

Since its discovery in 2011, ${ }^{1,2}$ the intronical GGGGCC expansion in non-coding region of chromosome 9 (Chromosome 9 Open Reading Frame 72, C9orf72) resulted responsible of most of the Frontotemporal Dementia (FTD) and it has been also described in a substantial number of apparently sporadic cases. ${ }^{3}$ The phenotypic range is associated with a considerable clinical heterogeneity, making early diagnosis difficult: most patients clinically show the behavioral variant of FTD (bvFTD) ${ }^{3}$ in which psychiatric symptoms are usually over-represented or predominate at onset leading to a diagnosis of a psychiatric disorder. ${ }^{4}$ Language disturbance like primary progressive aphasia (PPA) is a fairly rare phenotype of C9orf72 variation ${ }^{5}$ and deficits in episodic memory at onset, mimicking Alzheimer's disease, have been reported also in patients with C9orf72 mutations. ${ }^{6}$ Moreover, beyond these cognitive phenotypes, the FTD clinical spectrum could overlap with motor neuron disease (MND) and Parkinsonism. ${ }^{7,8}$ C9orf72-FTD patients also show a high variability in age at onset and disease duration, due to the incomplete and age-dependent penetrance of the mutation.

The clinical heterogeneity of this pathology motivates scientific research to identify specific clinical traits and biomarkers that can categorize accurately FTD subtypes. ${ }^{9}$

Regarding functional neuroimaging, a pathognomonic pattern of the C9orf72 mutation has not been described yet. In several studies, an extensive frontal lobe hypometabolism, which sometimes involves also to the posterior cingulate cortex and some temporal areas, was described, often consistent with the different clinical phenotypes. ${ }^{10-12}$

Several preclinical studies tried to evaluate the efficacy of biomarkers to identify earlier C9orf72 expansion carriers, to address pathology specific therapies, but contrasting results have been obtained. ${ }^{13-16}$

The study of presymptomatic stage of C9orf72 expansion carriers offers a peculiar temporal window in order to characterize changes in neuroimaging and serum biomarkers, similar to other neurodegenerative disease as Alzheimer's disease, ${ }^{17,18}$ that could be essential to start trials aimed to discover disease-modifying treatments.

Neurofilament light chains (NfL) have been evaluated as potential biomarkers to identify presymptomatic stages of C9orf72 expansion carriers, and it has been shown that they reflect disease severity and progression. Infact they reveal axonal damage, and they are increased in symptomatic but not in presymptomatic C9orf72 expansion carriers. A variety of studies examined $\mathrm{NfL}$ concentration in the cerebral spinal fluid (CSF) ${ }^{19}$ obtaining promising results as monitoring biomarkers of the disease, but there is a considerable interest in developing blood-based biomarkers because of their convenience and higher acceptability instead of lumbar puncture. Recently, a new essay based on the single-molecule array technique (Quanterix Simoa) ${ }^{20}$ allows a quantification of small concentration (down to subfemtomolar) of the analyte and is 25 -fold more sensitive than the previous electrochemiluminescence-based method. Through this technology, we detected serum NfL concentrations that would have been less sensitive using other standard methods as conventional sandwich ELISA and electrochemiluminescence-based method (ECL assay). ${ }^{21}$

Our goal was first to correlate the demographic, clinical and neuroimaging features of a cohort of C9orf72-associated FTD patients with a cohort of bVFTD sporadic patients to possibly characterize a specific profile that could lead to identify eligible subjects for the genetic analysis of C9orf72 gene. Our second aim was to evaluate, using Quanterix Simoa innovative technique, the $\mathrm{NfL}$ serum concentrations in presymptomatic and symptomatic C9orf72 expansion carriers and their role as biomarkers of disease activity and progression, respectively. This has further been investigated through a longitudinal evaluation of two C9orf72 expanded carriers families.

\section{2 | MATERIALS AND METHODS}

All the participants were identified at the Neurology Unit of Careggi Hospital, Florence, Italy, and they were selected following the Criteria of Rascovsky et al 2011. ${ }^{22}$

For the first aim of the study, three groups were created to correlate clinical and neuroimaging data: (a) 13 C9orf72-associated patients with FTD; (b) 25 bvFTD patients without C9orf72 expansion; (c) 25 cross-matched neurologically normal controls.

For the second goal, serum NfL concentration was analyzed in six C9orf72 FTD patients, six C9orf72 presymptomatic subjects, and seven healthy controls.

In addition, it has been possible to evaluate the serum NfL variation within 1 year in a subgroup composed of two unrelated families (with one symptomatic patient and two presymptomatic relatives in each family).

All the recruited patients were screened in order to evaluate the absence of other pathogenetic mutations in the progranulin (GRN) and microtubule-associated protein tau (MAPT) genes.

Finally, informed consent was obtained from each study participant and the study protocol was approved by the local ethic committee and conducted in accordance with the provisions of the Helsinki Declaration.

C9orf72 repeat expansion was searched using the repeat-primed PCR and automatic sequencing (3700 ABI PRISM Genetic Analyzer; Applied Biosystem), the characteristic stutter amplification pattern was considered as indication of pathogenetic repeat expansion ( $>30$ repeats).

All the coding exons and the intron/exon boundaries of the GRN and MAPT genes were polymerase chain reaction (PCR) amplified using primers designed with Primer3 software (http://bioinfo.ut.ee/ primer3-0.4.0/primer3/). The analysis was performed using high resolution melting analysis (HRMA) followed by direct sequencing of amplicons showing heteroduplex (310 ABI PRISM Genetic Analyzer; Applied Biosystems).

Measurement of serum $\mathrm{NfL}$ levels was performed in duplicated with Quanterix Simoa platform; longitudinal serum of presymptomatic and symptomatic C9orf72-FTD was collected at time 0 (TO) and after 12 months (T12). 
All patients underwent neurological physical examination, neuropsychological evaluation, and brain MRI.

Twenty-three patients performed cerebral 18FDG-PET (3/13 expanded C9orf72 and 20/25 unexpanded C9orf72). When allowed by disease progression, patients were followed up over time with clinical and neuropsychological follow-up (11/13 expanded C9orf72 and 21/25 unexpanded (9orf72). The average follow-up time was 16.5 months.

Clinical and neuropsychological evaluation was oriented to describe symptoms of onset that were categorized in groups, with partial reference to the Neuropsychiatric Inventory Questionnaire ${ }^{23}$ : behavioral disorders, changes in temperament, apathy/indifference, alterations in food behavior, memory deficits, attention deficit, language disorders, motor disorders (hyposthenia, fasciculation, hypertonicity, tremor, bradykinesia, walking, and balance disorders), and dysphagia. Neuropsychological evaluation was performed with MMSE, ${ }^{24}$ FABit, ${ }^{25} \mathrm{ADL},{ }^{26}$ and IADL. ${ }^{27}$

To evaluate the trend over time using clinical and neuropsychological parameters, we identified three groups:

- "Rapid progression" with presence of at least two of these characteristic: loss of autonomy within 1 year from the onset, loss of points in MMSE $\geq 10 / y$, loss of points in FABit > 3/y, immobilization syndrome or PEG placement within 3 years from the onset, and death within 5 years from the onset.

- "Average progression" with presence of at least two of these characteristic: loss of MMSE points $<10$ and $>2 / y$, loss of points to FABit $>1$ and $<3 / y$, loss of autonomy after $1-5$ years from the onset, and disease length between 5 and 12 years.

- "Slow progression" with presence of at least two of these characteristic: loss of points at MMSE $<2 / y$, loss of points at FABit $<1 / y$, loss of autonomy after more than 5 years from the onset, and disease length longer than 12 years.

MRI images (acquired with T1-weighted, T2-weighted and FLAIR sequences on a 1.5-T magnet and performed in all cases within 2 years of the onset of symptoms) were reviewed by two observers (an expert neuroradiologist and a neurologist) who attributed a score concerning:

- degree of frontal atrophy (0-3), through the GCA scale "global cerebral atrophy" ${ }^{28}$;

- degree of temporal atrophy (0-3), through the GCA scale;

- degree of mesial temporal atrophy (0-4) evaluated according to the MTA scale "medial temporal lobe atrophy"29;

- degree of leukoencephalopathy (0-4) according to the Fazekas scale ${ }^{30}$

Cerebral Positron Emission Tomography (PET) with 18F-FDG (fluorodeoxyglucose) was performed with a PET/CT Gemini TOF 16 scanner. Subjects received an $18 \mathrm{~F}-F D G$ injection (dose of $5 \mathrm{mCi} / 70 \mathrm{~kg}$ ) at rest in a poorly lit and silent room. Approximately 30 minutes after tracer injection, brain PET images were acquired.
We compared demographic characteristics, symptoms at the onset, neuropsychological evaluations, neuroimaging data, and the course of the disease between 13 patients with C9orf72 expansion and 25 patients with sporadic bvFTD.

\section{1 | Statistical analyzes}

Data were analyzed with SPSS software version 23 (IBM SPSS Statistics). The chi square and the Student $t$ tests were used for independent samples, where appropriate, to assess the existence of statistically significant differences between the two cohorts of subjects. To evaluate the possible value of single variables at the onset and follow-up in predicting the belonging to one of the two groups, the binary logistic regression test was used and the odds ratio was calculated for each significant variable in the model. $P$ values of $<.05$ were considered statistically significant

Statistical Parametric Mapping (SPM) was used to analyze PET images. Images were processed using SPM12 (Wellcome Department of Cognitive Neurology, Institute of Neurology, London). All images were spatially normalized to the reference template (Montreal Neurological Institute - MNI - McGill University, Quebec). A smoothing filter with $8 \mathrm{~mm}$ FWHM was applied to the standard images. Two sample t tests were used to compare patient groups with each other and with controls. Results were considered statistically significant at $P<.001$ unc. (cluster extent $>50$ )

\section{3 | RESULTS}

Demographic and clinical characteristics of the two compared groups of patients with FTD are showed in Table 1. Comparing the two groups, no differences in sex and Apolipoprotein $E$ (ApoE) genotype were found.

Focusing on the peculiar characteristics of patients with C9orf72 expansion, we found that the clinical phenotype most frequently associated with the mutation was bvFTD (7/13), followed by bvFTD with MND (6/13) and, in only one case, by PPA-MND. There was high variability of age at onset (from 41 to 73 years) and duration of disease (from 1 to 12 years and more). As compared to sporadic bvFTD, C9orf72 patients demonstrated significantly lower age at onset $(P=.004)$, higher familial form of the disease $(P=.13)$, higher numbers of affected relatives $(P=.0002)$, and higher FTD-MND symptoms $(P=.001)$. Regarding symptom at onset, more behavioral disorders (with a $p$ value at the limits of statistical significance), more motor disorders $(P<.001)$, greater dysphagia $(P=.006)$, and lower mnesic deficits $(P=.1)$ were associated significantly with C9orf72 FTD patients. Regarding progression of the disease, the subgroup of patients with C9orf72 expansion showed a higher rate of "Average" and "Rapid" progression $(P=.08)$ and a lower rate of "Slow" progression.

The linear regression model, which considers all the variables described above, is statistically significant $(P=.001)$, and it increases the percentage of patients correctly classified as belonging to the two categories (carrier of C9orf72 expansion or not carrier). 


\begin{tabular}{|c|c|c|}
\hline Variable & FTD-ORF $(n=18)$ & Sporadic FTD $(n=25)$ \\
\hline Gender (F - M) & $46.2 \%-53.8 \%$ & $40 \%-60 \%$ \\
\hline Age at onset $\mathrm{Y}$, mean (SD) & $60.5(9.5)^{*}$ & $69.4(8)$ \\
\hline Familial forms & $92.3 \%{ }^{*}$ & $52 \%$ \\
\hline $\begin{array}{l}\text { Number of relatives, mean } \\
\text { (SD) }\end{array}$ & $2.4(2)^{*}$ & $0.6(0.6)$ \\
\hline $\begin{array}{l}\text { APOE genotype }(3 / 2-3 / 3 \\
-3 / 4)\end{array}$ & $7.7 \%-69.2 \%-23.1 \%$ & $12.3 \%-66.7 \%-20.8 \%$ \\
\hline $\begin{array}{l}\text { Clinical Phenotype (FTD } \\
\text { - FTD/MND) }\end{array}$ & $53.8 \%-46.2 \%^{*}$ & $96 \%-4 \%$ \\
\hline Behavioral disorders (yes - no) & $61.5 \%-38.5 \%$ & $44 \%-66 \%$ \\
\hline $\begin{array}{l}\text { Change in temperament (yes } \\
\text { - no) }\end{array}$ & $69.2 \%-30.8 \%$ & $60 \%-40 \%$ \\
\hline Apathy (yes - no) & $38.5 \%-61.5 \%$ & $32 \%-68 \%$ \\
\hline $\begin{array}{l}\text { Alterations in food behavior } \\
\text { (yes - no) }\end{array}$ & $8 \%-92 \%$ & $20 \%-80 \%$ \\
\hline Memory deficits (yes - no) & $46 \%-54 \%$ & $68 \%-32 \%$ \\
\hline Attention deficit (yes - no) & $15.4 \%-84.6 \%$ & $32 \%-68 \%$ \\
\hline Language disorder (yes - no) & $23 \%-77 \%$ & $8 \%-92 \%$ \\
\hline Motor disorders (yes - no) & $21 \%-79 \%$ & $4 \%-96 \%$ \\
\hline Disphagia (yes - no) & $38.5 \%-61.5 \%$ & $4 \%-96 \%$ \\
\hline Frontal GCA (0 - 1 - 2 - 3) & $\begin{array}{l}0 \%-28.6 \%-42.9 \% \\
-28.6 \%\end{array}$ & $23.8 \%-38.1 \%-23.8 \%-14.3 \%$ \\
\hline Temporal GCA (0 - 1 - 2 - 3) & $\begin{array}{l}14.3 \%-28.6 \%-14.3 \% \\
-42.9 \%\end{array}$ & $20 \%-40 \%-30 \%-10 \%$ \\
\hline MTA dx (0 - 1 - 2 - 3 - 4) & $\begin{array}{l}28.6 \%-0 \%-28.6 \%- \\
42.9 \%-0 \%\end{array}$ & $\begin{array}{l}28.6 \%-23.8 \%-38.1 \%-4.8 \% \\
-4.8 \%\end{array}$ \\
\hline MTA sx (0 - $1-2-3-4)$ & $\begin{array}{l}28.6 \%-14.3 \%-28.6 \%- \\
14.3 \%-14.3 \%\end{array}$ & $\begin{array}{l}28.6 \%-28.6 \%-33.3 \%-4.8 \% \\
-4.8 \%\end{array}$ \\
\hline Fazekas score (0 - 1 - 2 - 3) & $50 \%-0 \%-33.3 \%-16.7 \%$ & $30 \%-40 \%-20 \%-10 \%$ \\
\hline $\begin{array}{l}\text { Evolution (slow - average } \\
\text { - fast) }\end{array}$ & $9.1 \%-36.4 \%-54.5 \%^{*}$ & $52.4 \%-33.3 \%-14.3 \%$ \\
\hline
\end{tabular}

TAB LE 1 Clinical and demographic characterization of patients with FTD

Abbreviations: ADL, activities of daily living; FABit, frontal assessment battery versione italiana; FoFTD/MND, fronto temporal dementia and motor neuron disease; GCA, global cerebral atrophy; MMSE, mini mental state examination; MTA, medial temporal lobe atrophy.

*Statistically significant different values between two groups with $P<.05$

All together these variables are able to predict significantly the presence of C9orf72 expansion with an accuracy of $89.5 \%$ (Table 2).

TABLE 2 Linear Regression model, the table lists only the variables that are statistically significant in predicting the belonging to C9orf72 group

\begin{tabular}{|lll|}
\hline Variable & $\boldsymbol{P}$ & OR \\
\hline Familiar aggregation, number of parents & $<.05$ & 9.9 \\
\hline FTD/MND pathology & $<.05$ & 28.5 \\
\hline Motor disturbances & $<.05$ & 1000 \\
\hline Dysphagia & .05 & 125 \\
\hline Rapid evolution & $<.05$ & 22 \\
\hline
\end{tabular}

Abbreviation: OR, odds ratio.
Analyzing neuroimaging data, we have observed that C9orf72mutated subgroup patients showed a trend of higher frontal atrophy than sporadic bvFTD. No differences were found in temporal atrophy and leukoencephalopathy's degree. FDG-PET analyzes indicated a more widespread hypometabolism in C9orf72 patients involving the entire frontal lobes and comprising premotor and motor areas and subcortical regions (striata and thalami) (Figures 1 and 2).

The analyzes of $\mathrm{NfL}$ levels revealed that in symptomatic C9orf72 patients, NfL concentrations were strongly elevated compared with the presymptomatic carriers which resulted similar to control subjects (Table 3). Moreover, the NfL values of three symptomatic patients included in the "Rapid progression group" were higher than the ones of the three symptomatic patients included in the "Average progression group" (NfL median value in the rapid progression group 
FIGURE 1 A, 3D render of clusters of significant hypometabolism in patients with sporadic bvFT, as compared to controls $(P<.001$, unc.). B, 3D render of clusters of significant hypometabolism in patients with C9orf72 mutation, as compared to controls $(P<.001$, unc.)
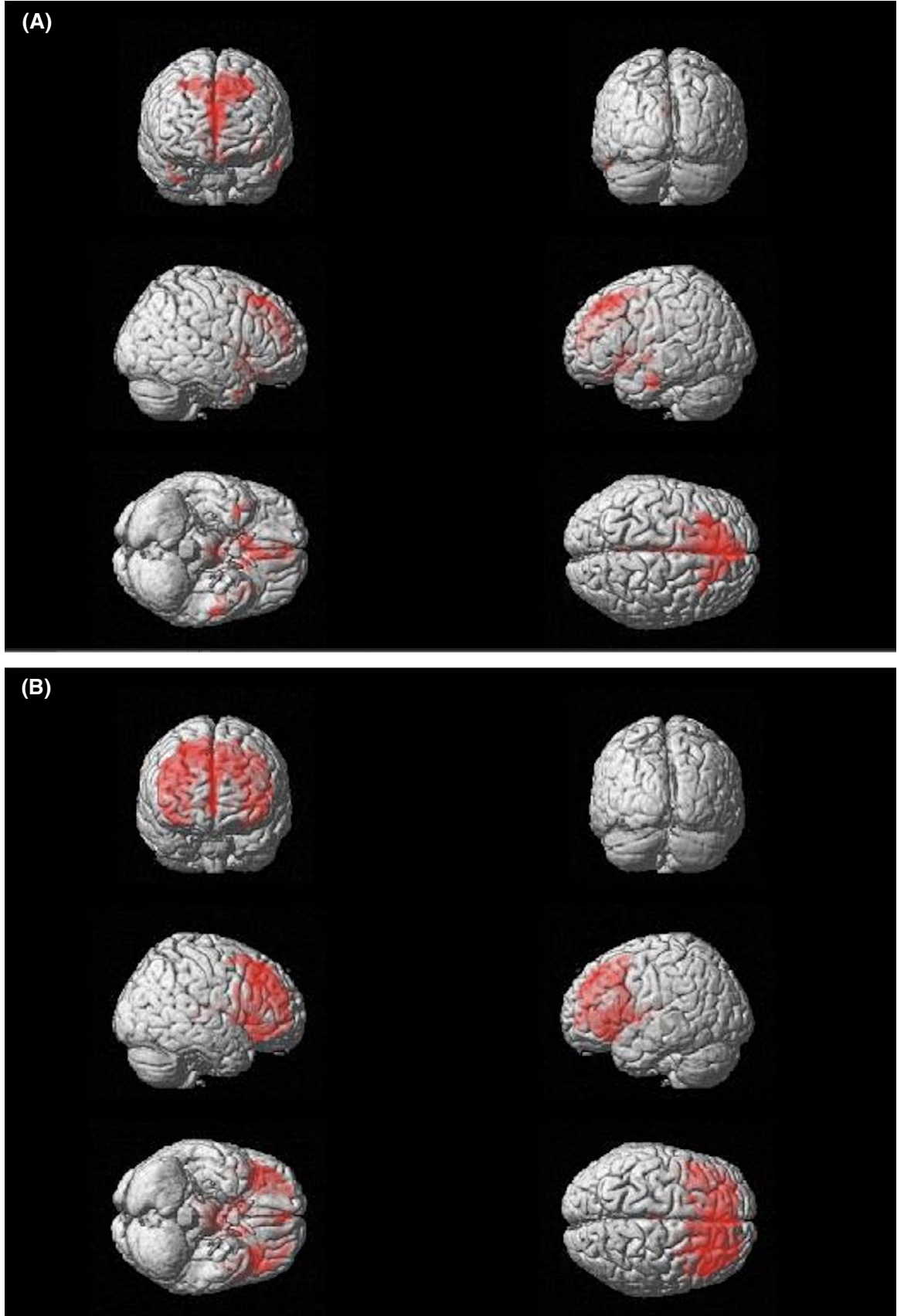

was $23.30 \mathrm{pg} / \mathrm{ml}$ respects to the median value in the average progression group of $14.64 \mathrm{pg} / \mathrm{ml}$. data not shown).

The longitudinal study of expanded families showed that serum $\mathrm{NfL}$ values in symptomatic C9orf72-FTD patients increased after 1 year; otherwise in presymptomatic carriers serum, NfL concentrations remained stable (Table 4).

\section{DISCUSSION}

In this study, we combined clinical, neuropsychological, neuroimaging, and biological features of 38 patients with FTD (13 with C9orf72-FTD and 25 with sporadic FTD without expansion in
C9orf72) to identify a specific signature of C9orf72 pathogenic expansion in FTD.

Our cohort of C9orf72 patients presents a high variability in age at onset, duration of the disease, familial forms, and number of affected family members, in accordance with the genetic epidemiology of Italian patients with FTD. ${ }^{31-36}$

These features reflect the incomplete and age-related penetrance of the mutation, that determines the high phenotypic heterogeneity characteristic of this pathology. As expected, there are no statistically significant differences in incidence among males and females and in the distribution of APOE e4 allele.

In our sample, the disease debuted very frequently with behavioral and psychiatric symptoms, not simply due to their high 


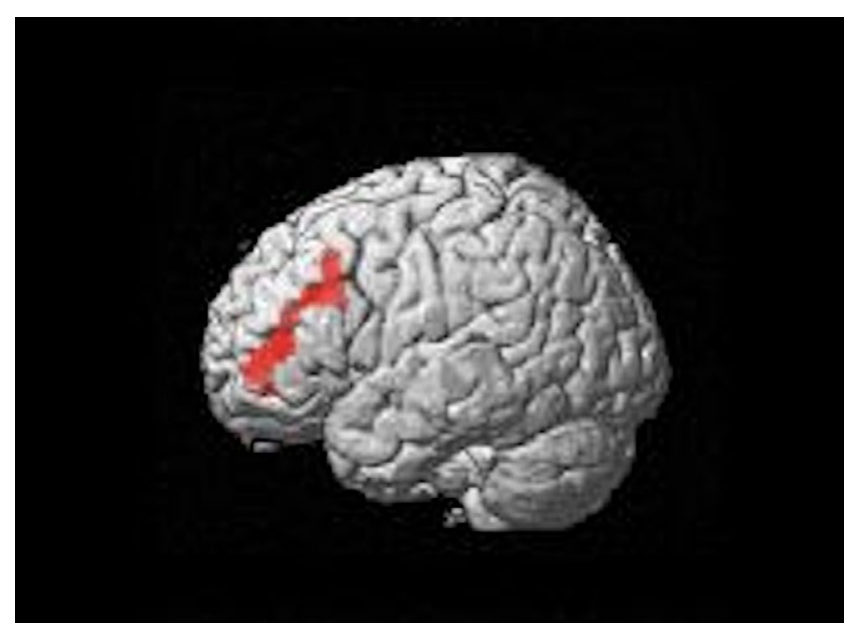

FIGURE 2 3D render of clusters of significant hypometabolism in patients with C9orf72-mutated patients compared to sporadic bvFTD patients $(P<.001$, unc. $)$

TABLE 3 Demographics, cognitive test scores, imaging measure and NfL concentration of Presymptomatic and Symptomatic carriers compared with non carriers

\begin{tabular}{|c|c|c|c|}
\hline & $\begin{array}{l}\text { Presymptomatic } \\
\text { carriers }\end{array}$ & $\begin{array}{l}\text { Symptomatic } \\
\text { carriers }\end{array}$ & $\begin{array}{l}\text { Control } \\
\text { subjects }\end{array}$ \\
\hline $\mathrm{N}$ & 6 & 6 & 7 \\
\hline Age. y, mean (SD) & $42,(9)$ & $64,(8)$ & $68,(7)$ \\
\hline Sex M/F & $4 / 2$ & $5 / 1$ & $4 / 2$ \\
\hline MMSE/30 & 29.0 & 21.0 & 30.0 \\
\hline Global CDR & $0(0-0)$ & $\begin{array}{c}0.5(0.5-1) \\
(n=4)\end{array}$ & $0(0-0)$ \\
\hline $\begin{array}{l}\text { Rate of frontal brain } \\
\text { atrophy (GCA) }\end{array}$ & 0.8 & 2.1 & 0.2 \\
\hline $\begin{array}{l}\text { Serum neurofila- } \\
\text { ment } \mathrm{pg} / \mathrm{ml} \text { (inter- } \\
\text { quartile range) }\end{array}$ & $1.99(1.68-2.65)$ & $\begin{array}{l}18.99 \\
(14.61-24.9)^{*}\end{array}$ & $\begin{array}{l}2.67 \\
(1.7-4.8)\end{array}$ \\
\hline
\end{tabular}

Note: (ANOVA one way).

${ }^{*} P<.0001$ between the three groups.

frequency in the bvFTD, but likely for the direct influence of the C9orf72 pathogenic expansion.

Other onset features that resulted significantly characteristic of our group of C9orf72 patients are young age, high number of affected relatives, FTD/MND symptoms and dysphagia. All these clinical characteristics together could lead to suspect with good accuracy (89.5\%) this mutation and thus direct the request of genetic analysis during the outpatient evaluation of the patients with FTD.

The analysis of the distribution of cerebral atrophy has shown a trend of greater frontal atrophy in the mutated patients, probably signifying a more serious involvement of this lobe. Congruously, the analysis of PET images revealed a cerebral hypometabolic pattern, that involves in particular the frontal lobes in C9orf72-expanded patients, which is compatible with psychiatric and movement disorders. These data suggest that these symptoms are maybe directly correlated with cortical dysfunction and not only with degeneration
TAB LE 4 Longitudinal study, serum NfL concentration in symptomatic C9orf72-FTD patients and in presymptomatic carriers after $1 \mathrm{y}$

\begin{tabular}{|c|c|c|c|c|c|}
\hline & $\begin{array}{l}\text { Time } \\
\text { from } \\
\text { onset }\end{array}$ & TO (pg/ml) & \multicolumn{2}{|c|}{$\mathrm{T} 12$ (pg/ml) } & T12 - T0 \\
\hline \multicolumn{6}{|l|}{ Family 1} \\
\hline Symtomatic & $8 \mathrm{mo}$ & 8.65 & \multicolumn{2}{|l|}{11.26} & 2.91 \\
\hline Presymptomatic\#1 & - & 2.26 & \multicolumn{2}{|l|}{2.78} & 0.52 \\
\hline Presymptomatic\#2 & - & 1.81 & \multicolumn{2}{|l|}{2.11} & 0.3 \\
\hline \multicolumn{6}{|l|}{ Family 2} \\
\hline Symtomatic & $13 \mathrm{mo}$ & 14.61 & \multicolumn{2}{|l|}{17.22} & 2.61 \\
\hline Presymptomatic\#1 & - & 1.49 & \multicolumn{2}{|l|}{1.92} & 0.43 \\
\hline \multirow[t]{2}{*}{ Presymptomatic\#2 } & - & 1.65 & 2.20 & & 0.55 \\
\hline & Mean TO & \multicolumn{2}{|c|}{ Mean T12 } & \multicolumn{2}{|c|}{ Mean T12 - T0 } \\
\hline Symtomatic $(n=2)$ & $11.63 \pm 4.21^{\mathrm{a}}$ & \multicolumn{2}{|c|}{$14.39 \pm 4.4^{b}$} & \multicolumn{2}{|c|}{$2.76 \pm 0.21^{\mathrm{a}}$} \\
\hline $\begin{array}{l}\text { Presymptomatic } \\
(\mathrm{n}=4)\end{array}$ & $1.83 \pm 0.33$ & \multicolumn{2}{|c|}{$2.25 \pm 0.37$} & 0.45 & \pm 0.11 \\
\hline
\end{tabular}

Note: (ANOVA one way).

${ }^{\mathrm{a}} P$ value $<.00001$

${ }^{\mathrm{b}} P$ value $=.003$.

of the second motor neuron. Besides, the relative spare of metabolic activity in precuneus and posterior cingulate cortex could correlate with the conservation of mnesic functions and MMSE scores observed in these patients even if in contrast with some data from literature ${ }^{37}$ in which anyway FDG-PET imaging was not performed.

Serum NfL levels are correlated with the progression of the disease, as showed in longitudinal samples, in which $\mathrm{NfL}$ values increased only in symptomatic carriers and not in presymptomatic subjects. A previous study on serum NfL levels confirmed that higher concentrations may reflect the intensity of the disease in patients with FTD and are associated with more rapid atrophy of the frontal lobes. ${ }^{38}$ Despite the very small number of our samples, which is a limit and that could influence the final conclusion, the NfL concentrations seem to be a very relevant biomarker useful to monitor the disease progression in clinical trials.

In conclusion, should clinicians now counsel all patients with sporadic FTD and test for C9orf72? The incomplete penetrance, the lack of satisfactory understanding of the concurrence of pathogenic mutations, the unknown correlation between length of the expansion and clinical phenotype with different prognoses, raises ethical questions in management of patient and his family members. ${ }^{39}$

Our principal aim was to identify peculiar characteristics of C9orf72-FTD patients, some of them already well described in literature, other ones less known, which in their whole could represent a useful tool to help the early diagnose this disease. Indeed, we can speculate that the metabolic signature combined with the clinical features could be used as helpful phenotypic predictors to direct the genetic counseling and the request of genetic analysis. Moreover, we confirmed that serum NfL evaluation could be a 
valid and easily obtainable biomarker to monitor the disease progression and severity.

\section{ACKNOWLEDGMENTS}

Italian Ministry of Health, Ricerca Finalizzata GR-2011-02347190, Fondo Ateneo 2017 Nacmias.

\section{CONFLICT OF INTEREST}

Authors declare no conflict of interest for this article.

\section{ORCID}

Silvia Bagnoli (iD https://orcid.org/0000-0002-4373-6611

\section{REFERENCES}

1. DeJesus-Hernandez M, Mackenzie I, Boeve B, et al. Expanded GGGGCC hexanucleotide repeat in noncoding region of C9ORF72 causes chromosome 9p-linked FTD and ALS. Neuron. 2011;72(2):245-256

2. Renton AE, Majounie E, Waite A, et al. A hexanucleotide repeat expansion in C9ORF72 is the cause of chromosome 9p21-linked ALSFTD. Neuron. 2011;72(2):257-268.

3. Majounie E, Renton AE, Mok K, et al. Frequency of the C9orf72 hexanucleotide repeat expansion in patients with amyotrophic lateral sclerosis and frontotemporal dementia: a cross-sectional study. Lancet Neurol. 2012;11(4):323-330.

4. Snowden JS, Rollinson S, Thompson JC, et al. Distinct clinical and pathological characteristics of frontotemporal dementia associated with C9ORF72 mutations. Brain. 2012;135(3):693-708.

5. Mahoney CJ, Beck J, Rohrer JD, et al. Frontotemporal dementia with the C9ORF72 hexanucleotide repeat expansion: clinical, neuroanatomical and neuropathological features. Brain. 2012;135(3):736-750.

6. Harms M, Benitez BA, Cairns N, et al. C9orf72 hexanucleotide repeat expansions in clinical Alzheimer disease. JAMA Neurol. 2013;70(6):736-741.

7. Cooper-Knock J, Hewitt C, Highley JR, et al. Clinico-pathological features in amyotrophic lateral sclerosis with expansions in C9ORF72. Brain. 2012;135(3):751-764.

8. Lesage S, Le Ber I, Condroyer C, et al. Parkinson's Disease Genetics Study Group. C9orf72 repeat expansions are a rare genetic cause of parkinsonism. Brain. 2013;136(2):385-391.

9. Rohrer JD, Nicholas JM, Cash DM, et al. Presymptomatic cognitive and neuroanatomical changes in genetic frontotemporal dementia in the Genetic Frontotemporal Dementia Initiative (GENFI) study: a cross-sectional analysis. Lancet Neurol. 2015;14(3):253-262.

10. Barkhof F, Fox NC, Bastos-Leite AJ, Scheltens P. Neuroimaging in Dementia. Berlin, Heidelberg: Springer; 2011.

11. Castelnovo V, Caminiti SP, Riva N, Magnani G, Silani V, Perani D. Heterogeneous brain FDG-PET metabolic patterns in patients with C9orf72 mutation. Neurol Sci. 2019;40(3):515-521.

12. Yokoyama JS, Rosen HJ. Neuroimaging features of C9ORF72 expansion. Alzheimer's Res Ther. 2012;4(6):45

13. Jiang J, Zhu Q, Gendron T, et al. Gain of toxicity from ALS/FTDlinked repeat expansions in C9ORF72 is alleviated by antisense oligonucleotides targeting GGGGCC-containing RNAs. Neuron. 2016;90(3):535-550.
14. Gendron TF, Chew J, Stankowski JN, et al. Poly(GP) proteins are a useful pharmacodynamic marker for C9ORF72-associated amyotrophic lateral sclerosis. Sci Transl Med. 2017;9(383):eaai7866.

15. Donnelly C, Zhang P-W, Pham J, et al. RNA toxicity from the ALS/ FTD C9ORF72 expansion is mitigated by antisense Intervention. Neuron. 2013;80(2):415-428.

16. Sareen D, O'Rourke JG, Meera P, et al. Targeting RNA foci in iPSCderived motor neurons from ALS patients with a C9ORF72 repeat expansion. Sci Transl Med. 2013;5(208):208ra149.

17. Weston P, Poole T, Ryan NS, et al. Serum neurofilament light in familial Alzheimer disease: a marker of early neurodegeneration. Neurology. 2017;89(21):2167-2175.

18. Kinnunen KM, Cash DM, Poole T, et al. Dominantly Inherited Alzheimer Network (DIAN) Presymptomatic atrophy in autosomal dominant Alzheimer's disease: a serial magnetic resonance imaging study. Alzheimers Dement. 2018;14(1):43-53.

19. Meeter L, Vijverberg EG, Del Campo M, et al. Clinical value of neurofilament and phospho-tau/tau ratio in the frontotemporal dementia spectrum. Neurology. 2018;90(14):e1231-e1239.

20. Rissin DM, Kan CW, Campbell TG, et al. Single-molecule enzymelinked immunosorbent assay detects serum proteins at subfemtomolar concentrations. Nat Biotechnol. 2010;28(6):595-599.

21. Kuhle J, Barro C, Andreasson U, et al. Comparison of three analytical platforms for quantification of the neurofilament light chain in blood samples: ELISA, electrochemiluminescence immunoassay and Simoa. Clin Chem Lab Med. 2016;54(10):1655-1661.

22. Rascovsky K, Hodges JR, Knopman D, et al. Sensitivity of revised diagnostic criteria for the behavioural variant of frontotemporal dementia. Brain. 2011;134(Pt 9):2456-2477.

23. Cummings JL, Mega M, Gray K, Rosenberg-Thompson S, Carusi DA, Gornbein J. The Neuropsychiatric Inventory: comprehensive assessment of psychopathology in dementia. Neurology. 1994;44(12):2308-2314.

24. Magni E, Binetti G, Bianchetti A, Rozzini R, Trabucchi M. MiniMental State Examination: a normative study in Italian elderly population. Eur J Neurol. 1996;3(3):198-202.

25. Appollonio I, Leone $\mathrm{M}$, Isella $\mathrm{V}$, et al. The Frontal Assessment Battery (FAB): normative values in an Italian population sample. Neurol Sci. 2005;26(2):108-116.

26. Katz S, Ford AB, Moskowitz RW, Jackson BA, Jaffe MW. Studies of illness in the aged. The index of ADL: a standardized measure of biological and psychosocial function. JAMA. 1963;185(12):914-919.

27. Lawton MP, Brody EM. Assessment of older people: self-maintaining and instrumental activities of daily living. Gerontologist. 1969;9(3 Part 1):179-186

28. Pasquier F, Leys D, Weerts JG, Barkhof F, Scheltens P. Inter- and intraobserver reproducibility of cerebral atrophy assessment on MR scans with hemispheric infarcts. Eur Neurol. 1996;36(5):268-272.

29. Duara R, Loewenstein DA, Potter E, et al. Medial temporal lobe atrophy on MRI scans and the diagnosis of Alzheimer disease. Neurology. 2008;71(24):1986-1992.

30. Fazekas F, Chawluk JB, Alavi A, Hurtig HI, Zimmerman RA. MR signal abnormalities at 1.5 T in Alzheimer's dementia and normal aging. Am J Roentgenol. 1987;149(2):351-356.

31. Capozzo R, Sassi C, Hammer MB, et al. Clinical and genetic analyses of familial and sporadic frontotemporal dementia patients in Southern Italy. Alzheimers Dement. 2017;13(8):858-869.

32. Bernardi L, Frangipane F, Smirne N, et al. Epidemiology and genetics of frontotemporal dementia: a door-to-door survey in southern Italy. Neurobiol Aging. 2012;33(12):2948.e1-2948.e10.

33. Bagnoli S, Piaceri I, Tedde A, et al. Progranulin genetic screening in frontotemporal lobar degeneration patients from central Italy. Cell Mol Neurobiol. 2012;32(1):13-16.

34. Borroni B, Archetti S, Alberici A, et al. Progranulin genetic variations in frontotemporal lobar degeneration: evidence for low 
mutation frequency in an Italian clinical series. Neurogenetics. 2008;9(3):197-205.

35. Seelaar H, Kamphorst W, Rosso SM, et al. Distinct genetic forms of frontotemporal dementia. Neurology. 2008;71(16):1220-1226.

36. Rohrer JD, Guerreiro R, Vandrovcova J, et al. The heritability and genetics of frontotemporal lobar degeneration. Neurology. 2009;73(18):1451-1456.

37. Irish M, Devenney E, Wong S, et al. Neural substrates of episodic memory dysfunction in behavioural variant frontotemporal dementia with and without C9ORF72 expansions. Neuroimage Clin. 2013;2:836-843.

38. Rohrer JD, Woollacott I, Dick KM, et al. Serum neurofilament light chain protein is a measure of disease intensity in frontotemporal dementia. Neurology. 2016;87(13):1329-1336.
39. Rohrer JD, Isaacs AM, Mizielinska S, et al. C9orf72 expansions in frontotemporal dementia and amyotrophic lateral sclerosis. Lancet Neurol. 2015;14(3):291-301.

How to cite this article: Lucidi G, Berti V, Piaceri I, et al. Clinical and neuroimaging profiles to identify C9orf72-FTD patients and serum Neurofilament to monitor the progression and the severity of the disease. Neurol Clin Neurosci. 2019;00:1-8. https://doi.org/10.1111/ncn3.12331 